\title{
Ultraperipheral Collisions at RHIC and LHC
}

\author{
Gerhard Baur \\ Institut für Kernphysik, Forschungszentrum Jülich, D-52425 Jülich, Germany
}

\begin{abstract}
A brief introduction to the physics of ultraperipheral collisions at collider energies is given. Photon-hadron (proton/ nucleus) and photon-photon interactions can be studied in a hitherto unexplored energy regime.
\end{abstract}

\section{Some features of Ultraperiph- eral Collisions (UPC)}

Photon-photon and photon-hadron interactions can also be studied in hadron-hadron collisions [1]. This may be surprising since in general such collisions are dominated by strong interactions between the hadrons. However, by choosing collisions with large impact parameter b (or, equivalently, small momentum transfer) one can suppress these strong interactions.

The time-dependent electromagnetic field of a fast moving charged particle can be thought of as a spectrum of (quasireal, or equivalent) photons [2, see Figure 1. The determination of the equivalent (or WeizsäckerWilliams) photon spectrum corresponding to a fast particle moving past an observer on a straight line path with impact parameter $b$ is a textbook example [3].

The probability $P(b)$ of a specific photonhadron reaction to occur in a collision with an impact parameter $b$ is given by $P(b)=$ $N(\omega, b) \sigma_{\gamma h}(\omega)$, where $\sigma_{\gamma h}$ is the corresponding photoproduction cross section. The equivalent photon spectrum can be calculated analytically, a useful approximation for qualitative considerations is

$$
N(\omega, b)=\frac{Z^{2} \alpha}{\pi^{2} b^{2}}
$$

for $\omega<\frac{\gamma}{b}$ and zero otherwise. The nuclear charge is given by $Z$, heavy ions have particularly high photon fluxes, however, this is partially offset by the lower ion-ion luminosities, as compared to the p-p case.

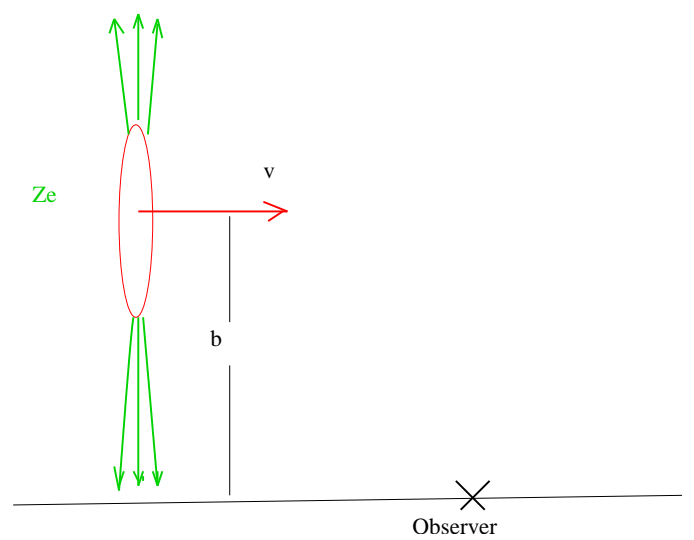

Figure 1: A fast charged particle moving on a straight line with impact parameter b causes a time-dependent electromagnetic field at the point of the observer. This field corresponds to a spectrum of equivalent photons.

The impact parameter $\mathrm{b}$ is restricted to

$$
b>b_{\text {min }} \sim R_{1}+R_{2}
$$

where $R_{1}$ and $R_{2}$ denote the sizes of the hadrons.

For heavy ion scattering the Coulomb parameter $\eta \equiv \frac{Z^{2} e^{2}}{\hbar v} \sim Z^{2} / 137$ is much larger than unity and it is in principle possible to determine the impact parameter by measuring the angle of Coulomb scattering. Whereas this is experimentally feasible at lower $(\sim G e V / A)$ energies [4], this angle is too small at collider energies. So one generally measures quantities integrated over all impact parameters. Too small impact parameters are recognized since the event is dominated by the violent strong interactions. 
The photon spectrum Eq. 1 extends up to a maximum photon energy given by

$$
\omega_{\max }=\frac{\gamma}{b_{\min }} .
$$

This energy is about $3 \mathrm{GeV}$ at $\mathrm{RHIC}(\mathrm{Au}-$ $\mathrm{Au}, \gamma \sim 100)$, and $100 \mathrm{GeV}$ at LHC (Pb-Pb, $\gamma \sim 3000)$ in the collider system.

\section{Multiphoton processes: possible trigger on UPC}

a

For heavy ions the probability of an electromagnetic interaction in ultraperipheral collisions is especially large, and multiphoton processes occur, see e.g. [5]. We mention $e^{+} e^{-}$pair production where the impact parameter dependent total pair production probability $P(b)$ is of order unity. Multiple pairs can be produced, however they may be hard to detect due to their low transverse momentum. The nuclear giant dipole resonance is excited with probabilities of order of one third. In Figure 2 one of the graphs is shown which leads to the electromagnetic production of a $\rho^{0}$ along with the excitation of the giant dipole resonance. These graphs can conveniently be evaluated in semiclassical or eikonal theories $[5]$.

The giant dipole resonance decays dominantly into a neutron. This neutron is detected in the forward direction and can serve as a trigger on UPC.

\section{UPC at RHIC}

The physics of UPC at RHIC and results from the STAR detector were covered by J. Seger in the session on photon- and electroweak boson physics, from HERA, RHIC and Tevatron to LHC.

A unique feature to photoproduction in hadron-hadron collisions is an interference effect [6]: a vector meson can be produced by a photon originating from either of the hadrons. It was shown in 6] that this interference effect leads to a reduction of the

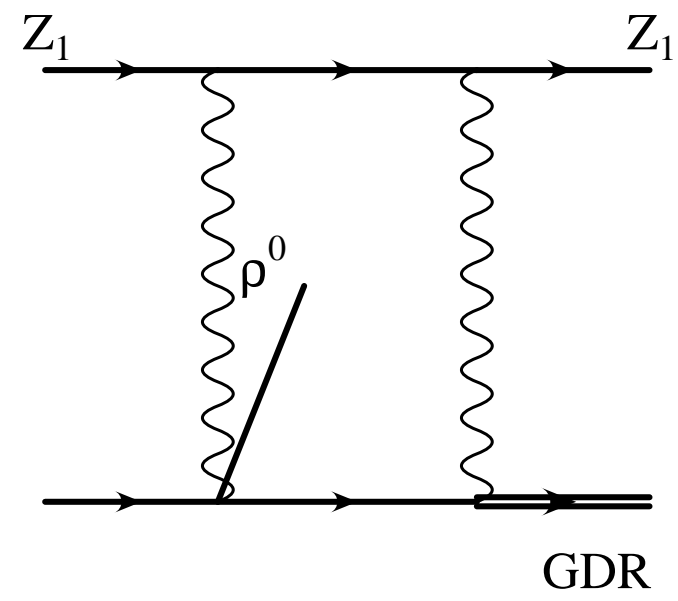

Figure 2: A graph contributing to the simultaneous production of a $\rho$-meson and the excitation of the giant dipole resonance (GDR).

transverse momentum spectrum of the vector mesons for small transverse momenta. Another theoretical approach [7] leads to very similar conclusions. (Preliminary) experimental results from STAR/RHIC indeed show a dip for small transverse momenta, see e.g. Ref. 8 .

\section{Opportunities for UPC at LHC}

The maximum photon energy scales linearly with the Lorentz factor $\gamma$, see eq. 3. This leads to a significant widening of the opportunities at LHC as compared to RHIC. A most promising area is low-x QCD studies. The experiments at HERA have shown that photoproduction processes provide a wellunderstood probe of the gluon density in the proton. At LHC, such processes could be extended to invariant $\gamma p$ energies exceeding the maximal HERA energy by a factor of 10. This would allow to use dijet (charm, etc.) production to measure the gluon density in the proton and/or nucleus down to $x \sim 3 \times 10^{-5}$. Ultraperipheral collisions 
would also allow one to study the coherent production of heavy quarkonia, $\gamma+A \rightarrow$ $J / \Psi(\Upsilon)+A$ at $x \lesssim 10^{-2}$, and to investigate the propagation of small dipoles through the nuclear medium at high energies, see Ref. [9], see also Refs. [10, 11. Dijet production via photon-gluon fusion is calculated in Ref. 12. Very large rates are obtained that will considerably extend the HERA x range.

In this session plans for studying UPC physics with heavy ions at the LHC were covered by J.Nystrand (ALICE), D.D'Enterria (CMS), and V. Pozdnyakov (ATLAS).

In addition to diffractive processes in proton-proton collisions at LHC also a rich program of proton-photon and photonphoton physics can be pursued, see Ref. [13. The photon flux is lower as compared to the heavy ion case due to the $Z^{2}$-factor, but this is at least partly compensated by higher beam luminosities. The photon spectrum is harder due to the smaller size as compared to the heavy ions, this leads to a lower value of $b_{\min }$ in Eq. 3 . Possibilities for electroweak physics and beyond were presented by S.Ovyn $(\gamma p)$ and T. Pierzchala $(\gamma \gamma)$ in this session. Tagging on photon energy by measuring the energy loss of the scattered protons in the forward detector TOTEM is an important feature. In this session J.Pinfold reported on photon-photon, photon-pomeron and double pomeron production at CDF.

A recent workshop on photoproduction at collider energies at ECT*/Trento was devoted to UPC, the mini-proceedings can be found in [14. The reviews [15, 16, 17, 18 and the most recent preprint 8] reflect the gradual progess of the field.

\section{Acknowledgments}

I would like to thank Frederic Kapusta for his kind invitation to this very pleasant and interesting conference at such a venerable place.

Photon 2007

\section{References}

[1] Slides:

http://indico.cern.ch/materialDisplay.py?

contribId=39\&sessionId $=16$

\&material Id=slides\&conf $I d=3841$

[2] E. Fermi, Z.Physik 29315 (1924)

[3] J.D. Jackson, Classical Electrodynamics (Wiley, New York, 1975)

[4] T. Aumann, Eur. Phys. J. A26 441 (2005)

[5] G. Baur et al., Nucl. Phys. A729 787 (2003)

[6] Spencer R. Klein and Joakim Nystrand Phys. Rev. Lett., 842330 (2000)

[7] K. Hencken, G. Baur, and D. Trautmann, Phys. Rev. Lett. 96012303 (2006)

[8] A. Baltz et al., arXiv:0706.3356

[9] Leonid Frankfurt, Mark Strikman, and Christian Weiss Annual Review of Nuclear and Particle Science $\mathbf{5 5} 403$ (2005)

[10] Yuri Ivanov, Boris Kopeliovich, and Ivan Schmidt, arXiv:0706.1532

[11] V. P. Gonçalves and M. V. T. Machado, arXiv:0706.2810

[12] Mark Strikman, Ramona Vogt, Sebastian White Phys. Rev. Lett. 96082001 (2006)

[13] Prospects for Diffractive and Forward Physics at the LHC CERN/LHCC 2006-039/G-124

[14] A. Baltz et al., arXiv: hep-ph/0702212

[15] C. A. Bertulani, and G. Baur, Phys. Rept. 163 299 (1988)

[16] F. Krauss, M. Greiner, G. Soff, Prog. Part. Nucl. Phys. 39503 (1997)

[17] G. Baur et al., Phys. Rept. 364359 (2002)

[18] Carlos A. Bertulani, Spencer R. Klein, and Joakim Nystrand, Annual Review of Nuclear and Particle Science 55271 (2005) 